\title{
Bilateraler Pneumothorax nach Akupunktur
}

\author{
Bilateral Pneumothorax Following Acupuncture
}

Autoren

Institute
L. Deterding', J. Bräunlich', G. Borte², H.-J. Seyfarth' ${ }^{1}$ H. Wirtz ${ }^{1}$

${ }_{1}^{1}$ Abteilung Pneumologie, Universitätsklinikum Leipzig AöR (Leiter: Prof. H. Wirtz)

${ }^{2}$ Klinik und Poliklinik für Diagnostische und Interventionelle Radiologie, Universitätsklinikum Leipzig AöR (Leiter: T. Kahn) eingereicht 9.9 .2014

akzeptiert 26.9.2014

\section{Bibliografie}

DOI http://dx.doi.org/

10.1055/s-0034-1390876

Pneumologie 2014; 68: 799-801

(c) Georg Thieme Verlag KG

Stuttgart · New York

ISSN 0934-8387

\section{Korrespondenzadresse \\ Lea Deterding}

Abteilung Pneumologie

Universitätsklinikum Leipzig AöR

Liebigstraße 20, Haus 4

04103 Leipzig

lea.deterding@uniklinik-leipzig. de

\section{Zusammenfassung \\ $\nabla$}

Die Akupunktur als Teilgebiet der traditionellen chinesischen Medizin ist fester Bestandteil alternativer Heilmethoden. Obwohl nur wenige Studien existieren, die einen therapeutischen Nutzen belegen, gewinnt sie in der westlichen Welt zunehmend an Bedeutung. Nicht zuletzt auch deshalb, weil sie als nebenwirkungsarm angesehen wird. Betrachtet man die Literatur, kann es jedoch, wenn auch selten, zu schwerwiegenden Komplikationen kommen.

Es wird über eine Patientin berichtet, die nach Akupunktur im Paravertebralbereich einen beidseitigen Pneumothorax erlitt. Diese Komplikation wurde nicht in die differenzialdiagnostischen Überlegungen einbezogen und auf diese Weise eine ohnehin lebensbedrohliche Situation zusätzlich kompliziert.

\section{Einleitung}

$\nabla$

Die Akupunktur ist eine beliebte alternative Heilmethode, die auch in der westlichen Welt zunehmend an Bedeutung gewinnt. Schwerwiegende Komplikationen werden nicht erwartet oder aber einer inkorrekten Punktionstechnik zugeschrieben. Daher wird die Akupunktur zunehmend häufiger als risikoarme Ergänzung zur sogenannten Schulmedizin eingesetzt. Als klassisches Beispiel ist die Therapie chronischer Schmerzen zu nennen. Auch im hier beschriebenen Fall sollten chronische Rückenschmerzen durch Akupunktur gelindert werden.

\section{Fallbeschreibung \\ $\nabla$}

Eine 64-jährige Patientin wird, vom Notarzt begleitet, in der zentralen Notfallaufnahme eines Universitätsklinikums vorgestellt. Kurze Zeit zuvor befand sie sich zur Behandlung chronischer

\section{Abstract \\ $\nabla$}

Acupuncture, a subsection of traditional Chinese medicine, plays an important role as an alternative healing method. Even though there is little proof of its efficacy, acupuncture is becoming more and more popular in the Western world, especially because it is considered almost free of side effects. However, severe complications may occur and have previously been described.

We will present a patient who suffered from bilateral pneumothoraces after acupuncture into the paravertebral area. This complication was not considered as a differential diagnosis thus even worsening the patient's life-threatening condition.

Rückenschmerzen in einer Akupunkturpraxis. Die Behandlung sollte die von der Patientin als unzureichend empfundene medikamentöse Schmerztherapie sinnvoll ergänzen. Die erfahrene Akupunkteurin plante fünf Sitzungen mit jeweils vier Punktionen im Bereich der thorakalen Wirbelsäule beidseits. Unmittelbar nach dem Einstechen der beiden oberen Nadeln bemerkte die Patientin punktuelle Schmerzen. Kurze Zeit später traten Ruhedyspnoe und Kaltschweißigkeit hinzu. Die Akupunkturnadeln wurden umgehend entfernt und der Rettungsdienst verständigt.

Dieser fand eine tachykarde (120/min), tachypnoeische (AF 30/min; transkutan gemessene $\mathrm{SaO} 2$ 60\%) und vigilanzgeminderte (GCS 9) Patientin vor. Das Atemgeräusch war beidseits abgeschwächt. Es ließen sich feuchte Rasselgeräusche auskultieren. Die nachgeforderte Notärztin zog differenzialdiagnostisch sowohl einen Myokardinfarkt als auch eine exazerbierte COPD in Betracht. Die Patientin erhielt Aspisol, Heparin, Prednisolon und Lasix. Bei hypertensiven Blut- 


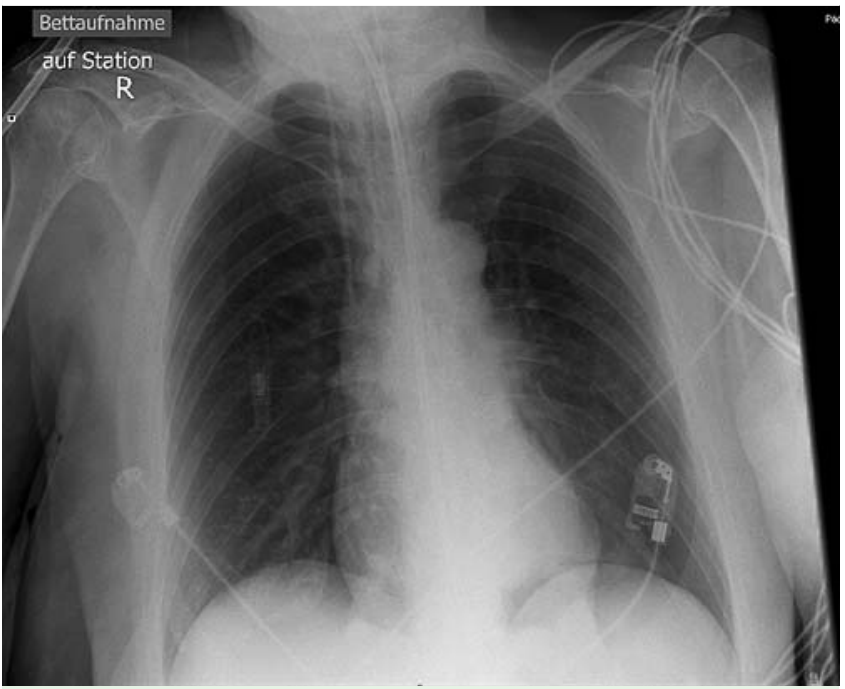

Abb. 1 Röntgen-Thorax im Liegen.

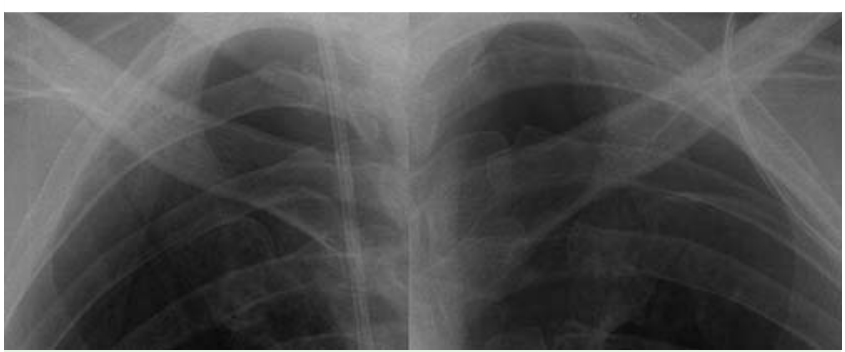

Abb. 2 Bilateraler apikaler Pneumothorax.

druckwerten verabreichte die Notärztin 2 Hub Nitro. Noch am Einsatzort musste die Patientin intubiert und kontrolliert beatmet werden.

Nach Ankunft in der Notfallaufnahme wurde die Patientin zunehmend hypoton $(97 / 50 \mathrm{mmHg})$. Das EKG zeigte neben einer weiterhin bestehenden Sinustachykardie einen Linksschenkelblock ohne weitere Auffälligkeiten. Die transthorakale Echokardiografie beschrieb eine anteroseptale Kinetikstörung mit Reduktion der Ejektionsfraktion auf $45 \%$. Die venöse Blutgasanalyse zeigte eine akute respiratorische Azidose mit einem $\mathrm{pH}$ von 7,031 und einem pCO2 von 95,2 mmHg. Die kranielle Bildgebung war unauffällig.

Noch ohne sichere Diagnose wurde die mittlerweile katecholaminpflichtige Patientin auf die internistische Intensivstation verlegt. Hier erfolgte auch das erste Röntgen-Thorax-Bild ( Abb. 1, Abb.2). Es zeigte bilaterale Pneumothoraces, welche umgehend mit zwei Pleurasaugdrainagen versorgt wurden. Bereits am Folgetag konnte die Patientin bei respiratorischer Stabilisierung extubiert werden. Die Drainagen wurden im Verlauf entfernt, und die Patientin konnte entlassen werden.

\section{Diskussion}

$\nabla$

Die Akupunktur ist ein Teilgebiet der traditionellen chinesischen Medizin. Dabei leitet sich der Begriff vom Lateinischen „acus“= Nadel und „pungere“=Stechen ab. Das Prinzip besteht in der positiven Beeinflussung eines gestörten Energieflusses mittels Stimulation definierter Triggerpunkte. Diese Energie, genannt

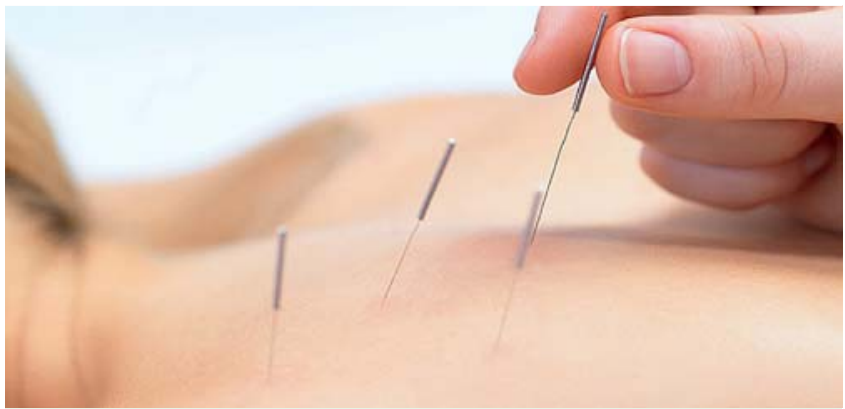

Abb.3 Akupunkturtechnik.

„Qi“, zirkuliert auf 14 symmetrischen Hauptmeridianen durch den Körper und steuert sämtliche Körperfunktionen. Ist der Energiefluss gestört, entstehen Krankheiten [1].

Die Geschichte der Akupunktur reicht bis ins dritte Jahrtausend vor Christus zurück. Ihre Theorie lieferte physiologische Erklärungsansätze und differenzierte sich somit eindeutig von unwissenschaftlichen Denkweisen. Durch zunehmenden wissenschaftlichen Erkenntnisgewinn im heutigen Sinne verlor die traditionelle chinesische Medizin im Laufe der Jahre an Bedeutung. Eine Art Renaissance erfuhr sie in den 1960er Jahren. Seither ist die Akupunktur ein fester Bestandteil des chinesischen Gesundheitssystems. Neuere Varianten wie Ohr-, Schädel- oder Handakupunktur entstanden [2].

Anwendung findet die Akupunktur hauptsächlich in der Therapie chronischer Schmerzen. An erster Stelle sind dabei Migräne, Rücken- und Gelenkbeschwerden zu nennen.

Es existieren jedoch nur wenige Studien, die einen therapeutischen Nutzen belegen. Eine klare Evidenz besteht z. B. für postoperatives, schwangerschaftsbedingtes und chemotherapieinduziertes Erbrechen. Geteilte Meinungen gibt es bei der Schmerzbehandlung [3]. In den German Acupuncture Trials, einer prospektiven und randomisierten Studie mit 3500 Probanden, zeigte sich die Akupunktur (Verum) in der Therapie chronischer Kopf-, Rücken- und Gelenkschmerzen überlegen. Allerdings bestand kein signifikanter Unterschied zwischen Verum und Akupunktur außerhalb der klassischen chinesischen Triggerpunkte (genannt „Sham“) ([4-7], ๑ Abb.3).

Mögliche Wirkmechanismen sind noch unzureichend verstanden. Eine zentrale Rolle könnte das analgetisch wirksame Adenosin spielen [8]. Sein Vorläufer, Adenosintriphosphat, wird durch mechanische oder elektrische Stimulation vermehrt freigesetzt. Beschrieben werden außerdem Aktivierungen des Hypothalamus, der Zirbeldrüse, Änderungen der Sekretion von Neurohormonen und Wirkungen auf den Blutfluss.

Alternative Heilverfahren wie die Akupunktur werden allgemein als nebenwirkungsarm angesehen. Die Fachliteratur beinhaltet jedoch einige, wenn auch insgesamt wenige, Fallberichte über schwerwiegende Akupunkturfolgen wie Pneumothoraces oder Perikardtamponaden. Letztere wurden laut Ernst et al. in der Fachliteratur insgesamt 26 Mal beschrieben; 14 dieser Fälle endeten tödlich [9]. Ebenfalls beschrieben wurde ein Todesfall nach Entstehung bilateraler Spannungspneumothoraces [10]. Die Inzidenz für solch schwerwiegende Komplikationen liegt in einer großen prospektiven Studie mit 190924 Probanden von Endres et al. aus dem Jahr 2004 jedoch nur bei 0,024\% [11]. Eine weitere, größere prospektive Studie mit 760000 durchgeführten Akupunkturen, ebenfalls aus dem Jahr 2004, beschreibt insgesamt sechs schwerwiegende Komplikationen, entsprechend 
einer Inzidenz von ca. 0,0008\%; zwei dieser Komplikationen waren Pneumothoraces [12]. Die weitaus häufigeren und auch weniger komplizierten unerwünschten Akupunkturfolgen beinhalten Schmerzen durch die Punktionsnadel, Blutungen im Bereich der Punktionsstelle und Müdigkeit [13]. In einer retrospektiven Studie wurden alle dem NHS (National Health System Großbritanniens und Nordirlands) über einen Zeitraum von drei Jahren gemeldeten unerwünschten Akupunkturfolgen entsprechend ihrer Häufigkeit sortiert: Häufig waren dabei im Körper zurückgelassene Nadeln (31\%), Schwindel (30\%) und Vigilanzminderungen (19\%). Der Pneumothorax wurde mit einer Häufigkeit von einem Prozent gemeldet [14].

Hinsichtlich des Pneumothorax stellt sich auch die Frage, ob eine Prädisposition des Patienten seine Entstehung begünstigte. Su et al. postulieren, dass vor allem schlanke, große Männer, Raucher, Patienten mit vorbeschriebenem Emphysem, Krebspatienten und solche mit Kortikoiddauertherapie ein erhöhtes Risiko für die Entstehung eines Pneumothorax nach Akupunktur haben [1]. Aufgrund der Seltenheit der Komplikationen wurde auch in unserem Fall zunächst kein Zusammenhang zwischen der Akupunkturbehandlung und oben beschriebener Symptomatik gesehen. Trotz typischer Symptome wie abgeschwächtes Atemgeräusch und Dyspnoe wurden andere Diagnosen für wahrscheinlicher gehalten; das zur Diagnose führende Röntgenbild erfolgte damit erst zu einem viel späteren Zeitpunkt. Ungewöhnlich ist, dass es in so kurzer Zeit zu Beatmungspflicht und Kreislaufdepression kam.

Zusammenfassend lässt sich also sagen, dass lebensbedrohliche Komplikationen zwar insgesamt selten sind, jedoch durchaus vorkommen können. Wichtig ist, an sie zu denken, sollte ein Patient Symptome im Zusammenhang mit Akupunktur entwickeln.

\section{Interessenkonflikt}

Die Autoren geben an, dass kein Interessenkonflikt besteht.

\section{Literatur}

$1 \mathrm{Su}$ JW, Lim CH, Chua YL. Bilateral pneumothoraces as a complication of acupuncture. Singapore Med J 2007; 48: e32

2 Akupunktur-Stiftung.de. Geschichte der Akupunktur. Im Internet: http://www.akupunktur-stiftung.de/akupunktur/sites/wissen/geschichte.htm [Stand: 06.10.2013]

3 (Anonym) NIH Consensus Conference. Acupuncture. JAMA 1998; 280 : $1518-1524$

4 Scharf HP, Mansmann U, Streitberger $K$ et al. Acupuncture and knee osteoarthritis: a three-armed randomized trial. Ann Intern Med 2006; 145: $12-20$

5 Haake M, Müller HH, Schade-Brittinger $C$ et al. German Acupuncture Trials (GERAC) for chronic low back pain: randomized, multicenter, blinded, parallel-group trial with 3 groups. Arch Intern Med 2007; 167: $1892-1898$

6 Diener HC, Kronfeld K, Boewing G et al. GERAC Migraine Study Group. Efficacy of acupuncture for the prophylaxis of migraine: a multicentre randomised controlled clinical trial. Lancet Neurol 2006; 5: 310-316

7 Endres HG, Böwing G, Diener HC et al. Acupuncture for tension-type headache: a multicentre, sham-controlled, patient-and observer-blinded, randomised trial. J Headache Pain 2007; 8: 306-314

8 Takano $T$, Chen $X$, Luo $F$ et al. Traditional acupuncture triggers a local increase in adenosine in human subjects. J Pain 2012; 13: 1215-1223

9 Ernst E, Zhang J. Cardiac tamponade caused by acupuncture: a review of the literature. Int J Cardiol 2011; 149: 287-289

10 Iwadate $\mathrm{K}$, Ito $\mathrm{H}$, Katsumura $\mathrm{S}$ et al. An autopsy case of bilateral tension pneumothorax after acupuncture. Leg Med (Tokyo) 2003; 5: 170-174

11 Endres HG, Molsberger A, Lungenhausen $M$ et al. An internal standard for verifying the accuracy of serious adverse event reporting: the example of an acupuncture study of 190,924 patients. Eur J Med Res 2004; 9: 545 - 551

12 Melchart $D$, Weidenhammer $W$, Streng $A$ et al. Prospective investigation of adverse effects of acupuncture in 97733 patients. Arch Intern Med 2004; 164: $104-105$

13 Saifeldeen K, Evans M. Acupuncture associated pneumothorax. Emerg Med J 2004; 21: 398

14 Wheway J, Agbabiaka TB, Ernst E. Patient safety incidents from acupuncture treatments: A review of reports to the National Patient Safety Agency. International Journal of Risk \& Safety in Medicine 2012; 24: $163-169$ 